\title{
A importância das teleconexões para a Previsão Sazonal
}

\author{
Importance of teleconnections for Seasonal Prediction
}

\author{
Tércio Ambrizzi ${ }^{1}$, Marcelo Alejandro Luna Sacco ${ }^{1}$ e Simone Erotildes Teleginski Ferraz ${ }^{2}$ \\ ${ }^{1}$ Departamento de Ciências Atmosféricas, Universidade de São Paulo, São Paulo, Brasil \\ ${ }^{2}$ Departamento de Física, CCNE, Universidade Federal de Santa Maria, RS, Brasil.
}

\begin{abstract}
Resumo
Este estudo analisa o desempenho das previsões por conjunto do modelo do European Center for Medium-Range Weather Forecast (ECMWF) utilizando a teoria linear das ondas, a fim de verificar seu desempenho e seus erros específicos. As composiçães sazonais da previsão foram para o período de 1981 a 2001 e a validação utilizou dados de reanálise do ECMWF (ERA40). Através das análises dos campos de $K_{5}$ (numero de onda total) e perfis meridionais em algumas longitudes, observou-se que a previsão sazonal dos possíveis guias de onda apesar de semelhantes aos dados da reanálise possuem pequenos deslocamentos latitudinais o que irá afetar o posicionamento dos guias de onda e, consequentemente, a propagação das ondas de Rossby. As diferenças obtidas através da técnica do ray tracing (Traçado de Raios) demonstram que o modelo não é capaz de reproduzir corretamente o estado médio básico da atmosfera na previsão sazonal. Dependendo da época ou do comprimento de onda o impacto da propagação remota pode ser muito diferente, uma vez que a fase e a energia das ondas estão deslocadas em relação aos dados observacionais e, portanto, a possibilidade de ocorrer chuva ou não em uma região específica (previsão) pode ser comprometida.
\end{abstract}

Palavras-chave: Teleconexões, Ray tracing, Modelo de previsão sazonal do clima.

\begin{abstract}
This study analyzes the performance of ensemble forecasts of the European Center for Medium-Range Weather Forecasting model (ECMWF) using the linear wave theory in order to check its performance and specific errors. Seasonal forecast composites for the period 1981 - 2002 were used as well as the ECMWF reanalysis (ERA40) for validation. The analysis of the total wavenumber (Ks) fields and some meridional profiles in selected longitudes indicated that the waveguide predicted by the model is similar to the reanalysis but there are some small but important differences, particularly related to the latitudinal displacement of the waveguides which in turn will affect Rossby wave propagation. Through the analysis of the ray tracing technique it is shown that the model is not able to correctly reproduce the atmosphere mean basic state in the seasonal forecast. Depending on the season or the wavelength propagation the remote impact may be very different, once the phase and energy of the wave will be displaced when compared to observations and therefore the possibility of, for instance, the model to predict rain (or no rain) in one specific region can be compromised.
\end{abstract}

Keywords: Teleconnection, Ray tracing, Seasonal Climate Model Forecast. 


\section{Introdução}

$\mathrm{O}$ clima global tem diversos padrões preferenciais de variabilidade que constituem o clima da superfície da terra. Alguns padrões refletem-se na circulação atmosférica, outros na oceânica, e muitos evoluem em função do acoplamento entre atmosfera e oceano. Todas estas preferências da variabilidade do clima são chamadas padrões de teleconexão. A palavra "teleconexão" significa conexão a distância. Sendo assim, podemos dizer que uma análise de teleconexão nos dá uma visão global da estrutura de ondas que foram geradas através de anomalias locais da circulação atmosférica e que influenciam regiões remotas. Desde o estudo pioneiro de Wallace e Gutzler (1981), que usaram médias mensais de altura de geopotencial em 500 hPa e pressão a nível do mar para produzir padrões de teleconexão para o inverno do Hemisfério Norte, vários outros estudos observacionais e numéricos tem se dedicado às análises das estruturas destes padrões, porém considerando ambos os hemisférios (p.ex., BLACKMON et al., 1984; HSU e LIN, 1992; HOSKINS e AMBRIZZI, 1993; AMBRIZZI et al., 1995, CAVALCANTI e AMBRIZZI 2009 e referências ali citadas).

Atualmente, todos os padrões de teleconexão são reconhecidos como modos preferenciais da variabilidade atmosférica, mas somente alguns deles são claramente identificados como sendo associados com forçante de Temperatura da Superfície do Mar (TSM). O trabalho de Horel e Wallace (1981) foi um dos primeiros a documentar a relação entre as anomalias de TSM equatoriais e o padrão do Pacífico/América do Norte (PNA). Grimm e Ambrizzi (2009) fizeram uma revisão completa dos diversos mecanismos que determinam a resposta extratropical à forçantes tropicais associadas com mudanças de TSMs e seus impactos sobre a América do Sul.

Apesar da natureza caótica da atmosfera, as previsões de longo prazo são possíveis até certo ponto, pelo fato de ter uma série de sistemas que apresentam variações em escalas de tempo mais lentas (estações e ou anos) e desta forma, possuindo um comportamento mais linear. Um bom exemplo deste tipo de sistema é o El Niño/Oscilação Sul (ENOS), que tem um ciclo temporal mais definido e influencia variáveis meteorológicas e suas flutuações em escala global. A relação entre o ENOS e sua influência remota na precipitação têm sido investigada por muitos autores (p.ex., STOECKENIUS, 1981; ROPELEWSKI e HALPERT, 1986, 1987; KILADIS e DIAZ, 1989). Em particular sobre a América do Sul, as teleconexões da fase quente do ENOS indicam déficit de precipitação ou mesmo secas na parte norte e nordeste do Brasil (p.ex., KOUSKY et al., 1984; UVO et al., 1998; ROPELWESKI e HALPERT, 1986, 1987; PEZZI e CAVALCANTI, 2001) e chuvas acima do normal sobre o sul do Brasil, norte da Argentina, Uruguai e Chile (p.ex., ACEITUNO, 1988; RAO e HADA, 1990, GRIMM et al., 1998, entre outros). Por outro lado, a fase fria do ENOS tem, em geral, mostrado chuvas com sinais opostos sobre as mesmas regiões (p.ex., GRIMM et al., 2000; ROPELEWSKI e HALPERT, 1989, AMBRIZZI et al. 2004). No entanto, deve ser lembrado que a variação inter-eventos ENOS pode gerar impactos diferenciados sobre as Américas conforme discutido em Magaña e Ambrizzi (2005).

Vários estudos têm enfocado a interação trópicos -extratrópicos, especialmente aqueles que envolvem a determinação da resposta atmosférica extratropical devido a forçante tropical associada a anomalias de TSM (veja TRENBERTH et al., 1998, GRIMM e AMBRIZZI 2009 e referências). Destes estudos, fica claro que anomalias de TSM como aquelas que ocorrem durante eventos quentes de ENOS, forçam anomalias de convecção e consequentemente subsidência de grande escala ligada ao ramo descendente da circulação de Hadley que resulta em perturbações nas ondas estacionárias planetárias climatológicas e correntes de jatos associados (AMBRIZZI et al. 2004; FREITAS e AMBRIZZI 2012). Estas perturbações têm certamente um impacto nos padrões globais de teleconexões.

Vários estudos já mencionaram que por causa das teleconexões associadas ao ENOS o skill dos modelos numéricos para a previsão sazonal é grande em algumas regiões do globo e em particular, na América do Sul (p.ex., FOLLAND et al. 2001; CAVALCANTI et al. 2002; MARENGO et al. 2003; MOURA e HASTENRATH 2004; COELHO 2005; COELHO et al. 2005, 2006). Uma boa qualidade da previsão sazonal do clima é importante para os governos locais tomarem ações de mitigação a fim de minimizar perdas econômicas e humanas geradas por eventos climáticos extremos como o ENOS e outros. Em particular, para a América do Sul, estes tipos de previsões são úteis para: a defesa civil, agricultura, pesca e gerenciamento de reservatórios. O Brasil, sendo o maior e mais populoso país do continente sul americano, gera mais de $90 \%$ de sua energia através de hidroelétricas. Este aspecto, principalmente ao longo dos últimos anos onde a variabilidade da precipitação tem aumentado em função de forçantes antrópicas ou não (p.ex., DRUMOND e AMBRIZZI 2005; MARENGO et al. 2011), enfatiza ainda mais a necessidade de se produzir uma previsão sazonal de chuvas de boa qualidade, o que certamente beneficiaria o governo brasileiro no gerenciamento e ações referentes a um controle mais eficiente da produção energética nacional.

Baseado nos conhecimentos já adquiridos dos padrões de teleconexão atmosférica que afetam o clima do Hemisfério Sul e em particular da América do Sul, o principal objetivo deste estudo é avaliar o desempenho de previsões sazonais por conjunto (ensembles) do modelo do centro europeu (European Centre for Medium-Range and Weather Forecasts - ECMWF) para vários anos através do uso da teoria linear de ondas. Saber onde e como os modelos estão falhando na representação dos principais padrões de circulação que afetam o continente sul americano, poderá auxiliar em seu desenvolvimento e desta 
forma na melhoria das previsões sazonais.

\section{Dados e metodologia}

\subsection{Dados}

Dados mensais do conjunto da reanálise do ECMWF (ERA 40) para o período de 1981 a 2001 serão utilizados para comparar com as simulações da previsão sazonal produzidas pelo modelo acoplado oceano-atmosfera do ECMWF (Integrated Forecast System - versão 3) que constituem um conjunto mensal contendo em média 11 membros com previsões de seis meses cada um. Para maiores detalhes do modelo acoplado veja Anderson et al. (2007).

A verificação da qualidade das previsões do modelo foi feita a partir da separação de cada um dos 20 anos de dados em estações de verão (Dezembro, Janeiro, Fevereiro; DJF) e inverno (Junho, Julho, Agosto; JJA) para a previsão sazonal do modelo para cada um dos 11 membros, identificado daqui em diante por 3MES (previsão de três meses). Foram analisados não somente as médias sazonais da previsão do conjunto mas também a análise de dispersão dos membros. Para obter os erros nas representações do estado básico da atmosfera como variabilidade espacial e intensidade do vento zonal em $200 \mathrm{hPa}$ foi feito a diferença entre a resposta do modelo e o dado considerado como padrão, que neste caso é o ERA40. Perfis meridionais e variações longitudinais do vento em latitudes selecionadas em 30 e $50^{\circ} \mathrm{S}$ para os 11 membros e o ERA40, assim como a média das composições para o numero de onda total $\left(\mathrm{K}_{5}\right)$ e perfis latitudinais em $120^{\circ} \mathrm{E}, 180^{\circ}, 120^{\circ} \mathrm{W}, 65^{\circ} \mathrm{W}$ também serão mostrados. Neste ultimo caso, análises de traçado de raios ("Ray Tracing") sobre o Hemisfério Sul foram desenvolvidas e são descritas na próxima seção.

\subsection{Descrição da metodologia}

\subsubsection{Teoria da propagação de ondas de Rossby uma breve revisão}

A relação de dispersão para ondas barotrópicas de Rossby embebidas em um escoamento que apresenta vento médio zonal $\bar{U}$, cuja perturbação pode ser escrita na forma $\exp [\mathrm{i}(\mathrm{kx}+\mathrm{ly}-\omega \mathrm{t})]$ :

$$
\omega=\overline{\mathrm{U}} \mathrm{k}-\frac{\beta_{*} \mathrm{k}}{\mathrm{K}^{2}}
$$

onde

$$
\beta_{*}=\frac{\mathrm{df}}{\mathrm{dy}}-\frac{\partial^{2} \overline{\mathrm{U}}}{\partial \mathrm{y}^{2}}
$$

é o gradiente meridional de vorticidade absoluta $\mathrm{e}$ $\mathrm{K}=\left(\mathrm{k}^{2}+\mathrm{l}^{2}\right)^{1 / 2}$ é o número de onda total, sendo $\mathrm{k}$ e $\mathrm{l}$ os números de onda zonal e meridional, respectivamente. $O$ vetor velocidade de grupo é dado por:

$$
\mathrm{c}_{\mathrm{g}}=\left(\mathrm{u}_{\mathrm{g}}, \mathrm{v}_{\mathrm{g}}\right)=\left(\frac{\partial \omega}{\partial \mathrm{k}}, \frac{\partial \omega}{\partial \mathrm{l}}\right)=\left(\mathrm{c}+\frac{2 \beta \mathrm{k}^{2}}{\mathrm{~K}^{4}}, \frac{2 \beta \mathrm{kl}}{\mathrm{K}^{4}}\right)
$$

onde $c=\omega / k$ é a velocidade de fase na direção $x$ (zonal).

Ondas estacionárias de Rossby apresentam $\omega=0$, e portanto $\mathrm{c}=0$. Logo, o número de onda total para ondas estacionárias $\left(\mathrm{K}_{\mathrm{s}}\right)$ pode ser escrito da seguinte forma:

$$
\mathrm{K}=\mathrm{K}_{\mathrm{s}}=\left(\frac{\beta_{*}}{\overline{\mathrm{U}}}\right)^{1 / 2}
$$

Dessa forma, tais ondas somente são possíveis perante a escoamentos de oeste ( $\overline{\mathrm{U}}$ positivo) e em regiões que apresentam $\beta^{*}$ positivo.

\subsection{Traçado de raios ou "Ray Tracing"}

Análise de padrões de teleconexões atmosféricas forçadas por fontes de calor será realizada através da teoria da propagação de ondas de Rossby utilizando-se uma técnica conhecida como Traçador de Raios ("Ray tracing").

Estudos prévios (p.ex. HOSKINS e KAROLY 1981) mostraram que a teoria linear de ondas pode explicar algumas das variabilidades atmosféricas de baixa frequência observadas. Através da utilização de um modelo numérico simplificado alimentado por um escoamento básico climatológico referente ao verão austral (DJF), Hoskins e Ambrizzi (1993) estudaram a trajetória preferencial seguida pelas ondas de Rossby. Para as análises de dispersão de ondas de Rossby eles derivaram a expressão que fornece o raio de curvatura referente ao caminho preferencial da propagação da energia presente nestas ondas que é dado por:

$$
\mathrm{r}=\frac{\mathrm{K}_{\mathrm{s}}^{2}}{\left(\mathrm{k} \frac{\mathrm{dK}}{\mathrm{dy}}\right)}
$$

Assim, através da distribuição global de Ks é possível identificar as trajetórias preferenciais de propagação das ondas de Rossby para vários escoamentos médios. $\mathrm{O}$ acordo entre os raios de curvatura e soluções numéricas do modelo barotrópico linearizado indicam que as características essenciais da propagação das ondas de Rossby são representadas pelos raios. Os resultados das 
soluções numéricas são conhecidos como "ray tracing".

A teoria de ondas estacionárias de Rossby foi utilizada com sucesso para explicar alguns padrões de teleconexões observados (HSU e LIN, 1992; AMBRIZZI et al., 1995). Entretanto, Karoly (1983) e alguns anos depois Yang e Hoskins (1996) apontaram a possível existência de algumas diferenças com relação a ondas de Rossby de baixa frequência, particularmente em suas velocidades de fase. Por simplicidade apenas ondas de Rossby referentes ao caso estacionário $(\omega=0, \mathrm{c}=0)$ serão analisadas.

\section{Resultados}

Conforme descrito anteriormente, a principal hipótese a ser investigada aqui é se o modelo de previsão consegue reproduzir os padrões gerais de propagação de ondas na atmosfera. Isso será feito através das análises do estado básico, particularmente o vento zonal, pois a variabilidade dos jatos atmosféricos influencia no direcionamento da propagação das ondas planetárias, agindo como guias. Consequentemente, se o modelo não for capaz de posicionar corretamente estes guias, a resposta final do mesmo poderá ser incorreta, colocando as ondas de Rossby estacionárias em regiões distintas da observação e, portanto, errando padrões meteorológicos locais, particularmente chuva.

\subsection{Características dos jatos em 200 hPa no hemisfério sul}

O jato subtropical no verão (Fig.1a) encontra-se em sua posição extrema sul entre 40 e $60^{\circ} S$ e tem um núcleo de $35 \mathrm{~m} / \mathrm{s}$ ao sul do oceano Índico. Possui uma abrangência que vai desde $60^{\circ} \mathrm{W}$ até $140^{\circ} \mathrm{E}$ e é zonalmente simétrico. $\mathrm{O}$ 3MES (Fig.1b) consegue reproduzir os principais campos espaciais observados no ERA40 tendo velocidades do vento semelhantes nas mesmas posições. No entanto, o modelo intensifica a área que abrange o vento zonal ao sudeste da Argentina com intensidades de $30 \mathrm{~m} / \mathrm{s}$. No inverno (fig.1c) o jato subtropical encontra-se em sua posição extrema norte em torno de $30^{\circ}$ S, e alcança sua máxima intensidade, com uma velocidade do vento acima de $50 \mathrm{~m} / \mathrm{s}$ ao leste da Austrália. O 3MES (fig.1d) consegue reproduzir a posição e orientação deste máximo de vento, mas mostra outro núcleo no setor Oeste da Austrália. Sobre América do Sul pode-se observar que um vento zonal de $35 \mathrm{~m} / \mathrm{s}$ atinge a zona leste de Uruguai na observação (fig.1c), no entanto, o modelo estende a área de $35 \mathrm{~m} / \mathrm{s}$ entre 20 e $40^{\circ}$ de latitude Sul, com uma simetria mais zonal do que aquela obtida no ERA40.

Em uma primeira análise pode-se concluir que a previsão sazonal para DJF e JJA é relativamente boa e reproduz de forma muito similar os padrões dos jatos hemisféricos. Apesar desta aparente semelhança existem pequenas diferenças que em princípio podem ser importantes na manutenção da propagação das ondas de Rossby. A fim de identificar onde o modelo superestima ou subestima os campos espaciais do vento zonal no HS foram determinadas as diferenças entre os dados do 3MES e ERA40, enfatizando assim o viés do modelo (Fig.2). Nota-se que durante o verão a previsão tende a subestimar a intensidade do vento zonal em praticamente toda a média latitude do HS (Fig.2a), enquanto que durante o inverno (Fig.2b) o jato polar é subestimado mas o jato subtropical é mais intenso, inclusive sobre a América do Sul. Esta diferença em intensidades pode afetar a propagação das ondas de Rossby, confinando-as

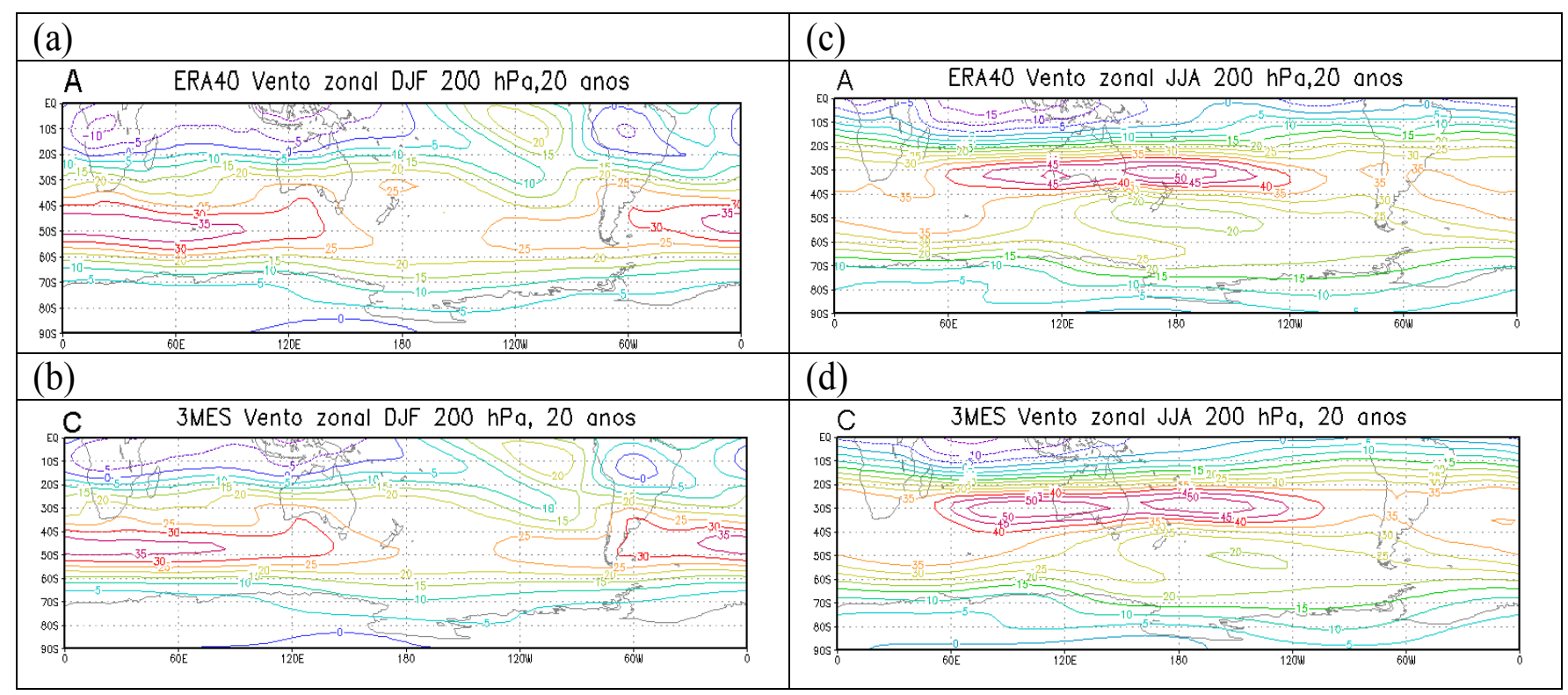

Figura 1: Composição de 20 verões (DJF) (a) e invernos (JJA) (c) para o vento zonal no nível de 200 hPa. para o ERA40 e (b) e (d) a previsão sazonal 3MES. O contorno de velocidade é em m/s. 


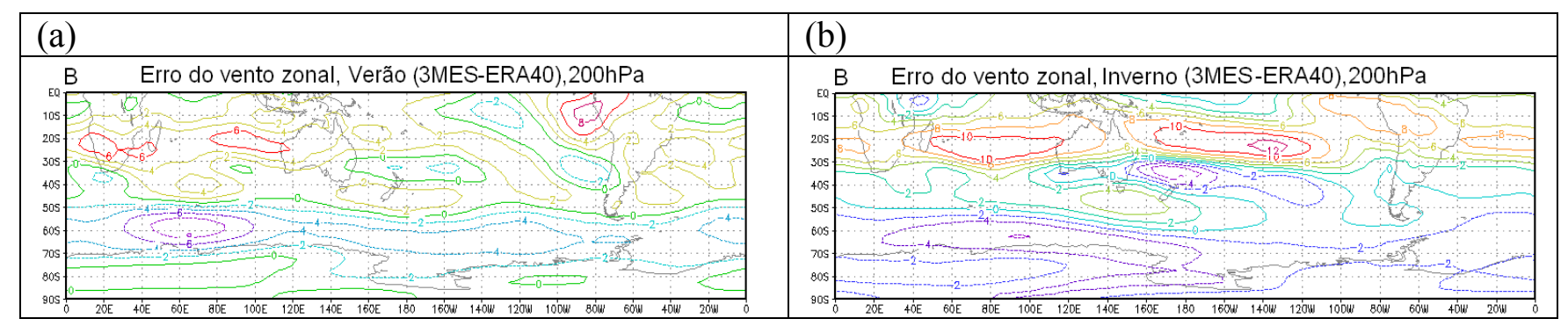

Figura 2: - Viés do vento zonal em 200 hPa (3MES-ERA40) para a estação de verão (a) e inverno. Contornos a cada $2 \mathrm{~m} / \mathrm{s}$ e linhas tracejadas corresponde a valores negativos.

mais facilmente nos subtrópicos, inclusive direcionando para a parte sudeste da América do Sul. Esta possibilidade será explorada mais adiante através das analises dos campos de número de onda estacionário.

\subsection{Corte longitudinal no campo do vento zo- nal em $200 \mathrm{hPa}$}

Com as 11 saídas do modelo ECMWF, pretende-se avaliar a dispersão entre os membros e comparar sua previsão sazonal com os dados do ERA40. Das composições sazonais foi escolhida a latitude $30^{\circ} S$ para realizar o corte longitudinal tendo em vista que jato subtropical esta presente na maior parte do ano (outono, inverno e primavera). A cor preta na Figura 3 corresponde ao valor climatológico obtido do ERA40, e as outras cores representam os 11 membros para cada estação do ano (DJF e JJA).

Como discutido anteriormente na análise do vento zonal para o verão (Fig.1a) o jato subtropical tem uma velocidade menor em comparação ao inverno (Fig.1b), no entanto, a previsão sazonal de verão reproduz relativamente bem a variação do fluxo zonal em $30^{\circ}$ S, embora superestime seus valores ao longo das longitudes, estando mais próximo do ERA40 sobre a América do Sul
(Fig.3a). No inverno (Fig.3b) quando o jato subtropical tem o máximo valor sobre Austrália, pode se notar uma grande diferença na posição do núcleo a oeste da Austrália onde a $3 \mathrm{MES}$ atinge o máximo em torno de $90^{\circ} \mathrm{E}$, com valores médios dos membros de $54 \mathrm{~m} / \mathrm{s}$, sendo que o valor obtido pelo ERA40 é de $46 \mathrm{~m} / \mathrm{s}$ para a mesma região. Depois da longitude de $180^{\circ}$ a oeste até por volta de 70 o $W$, o modelo superestima os ventos deslocando para leste os picos de máximos e mínimos.

É interessante notar pelos resultados acima que a embora a previsão sazonal do vento seja superestimada e os picos dos valores se mostrem deslocados quando comparados com o ERA40, para a latitude escolhida, a dispersão entre os membros do modelo é relativamente baixa, o que certamente aumenta o grau de confiabilidade no mesmo.

\subsection{Média latitudinal do vento zonal em $200 \mathrm{hPa}$}

A análise da variabilidade latitudinal do vento zonal em altos níveis mostra que durante a estação de inverno (Fig.4b) o corrente de jato é mais intensa do que no verão, conforme discutido anteriormente. No entanto, nesta estação são observados dois máximos, o primeiro s próximo a $30^{\circ} \mathrm{S}$ e o outro em $55^{\circ} \mathrm{S}$ com valores de 41

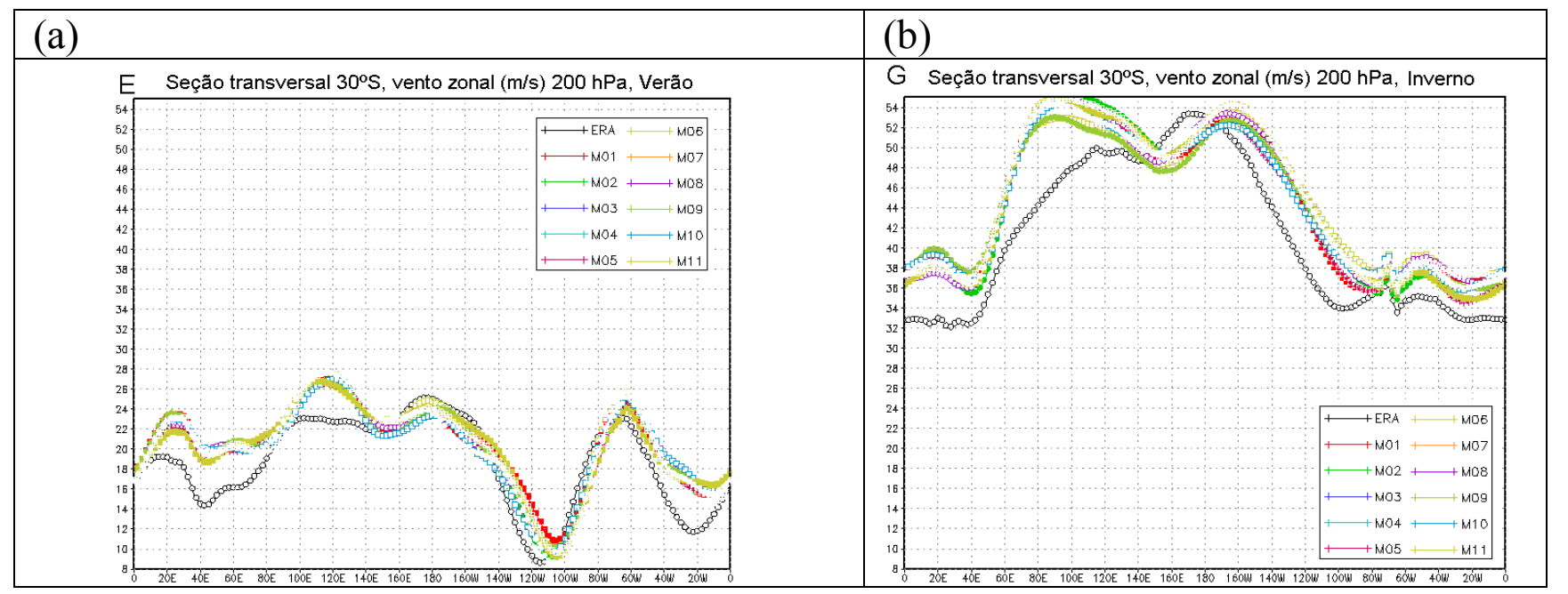

Figura 3: Corte longitudinal em $30^{\circ} \mathrm{S}$ (vento zonal - m/s), entre as composições sazonais de Verão (a) e Inverno (b) para o ERA40 (cor preta) e para cada um dos 11 membros do modelo representado por diferentes cores. 


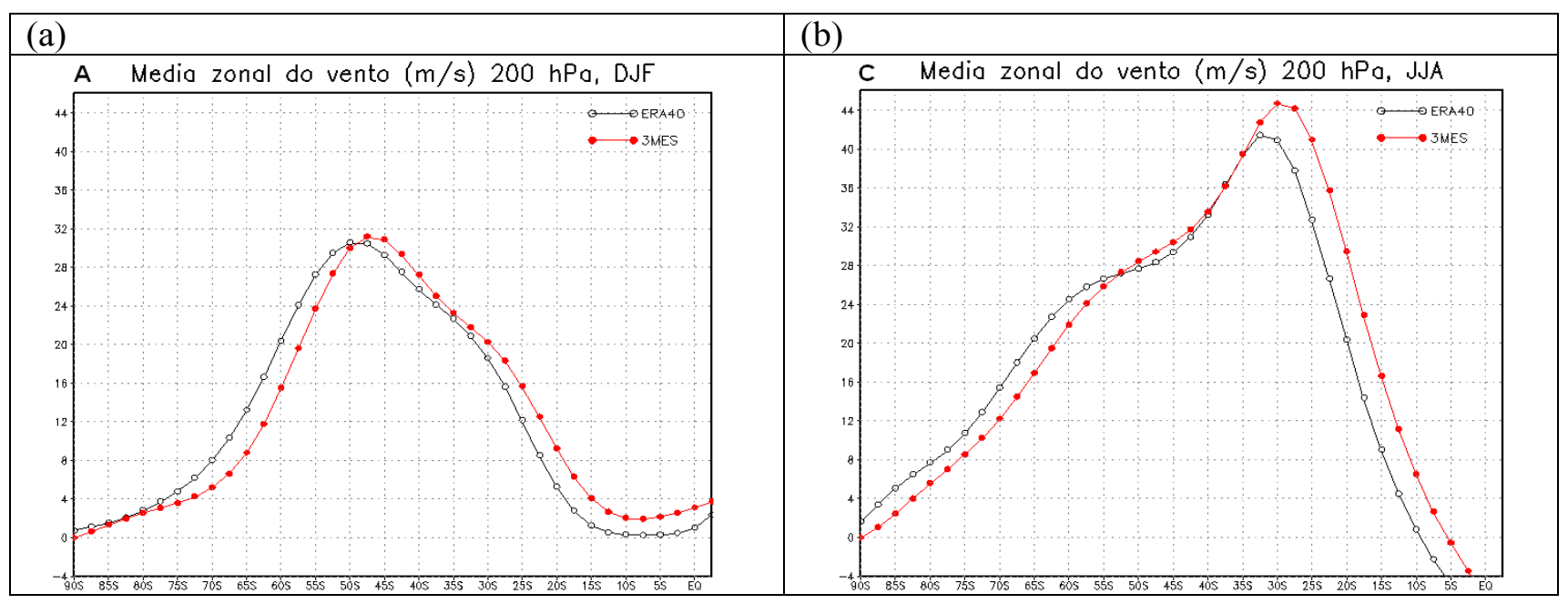

Figura 4: Média zonal do vento (m/s) em 200 hPa para o Hemisfério Sul baseado no ERA40 (linha preta) e Previsão Sazonal (3MES) para as estações de (a) verão e (b) inverno.

e $26 \mathrm{~m} / \mathrm{s}$, respectivamente. A previsão superestima o máximo em $30^{\circ} \mathrm{S}$ em $4 \mathrm{~m} / \mathrm{s}$ e o desloca para mais baixas latitudes. Para o verão (fig.4a) observa-se um máximo em aproximadamente $50^{\circ} \mathrm{S}$ com um valor de $30 \mathrm{~m} / \mathrm{s}$, onde apesar do modelo conseguir reproduzir este valor, ele também mostra um deslocamento do fluxo médio zonal em direção ao equador. Através da Fig.4 fica evidente que os guias de onda gerados pelo modelo, baseados no posicionamento dos máximos do vento zonal (HOSKINS e AMBRIZZI 1993) são deslocados de sua posição esperada e, portanto afetariam a propagação das ondas de Rossby. Este resultado fica mais claro na próxima seção onde as análises do Ks indicam os caminhos preferenciais das ondas.

\subsection{Análise do Ks para as estações de verão e inverno}

A Fig.5a corresponde aos campos espaciais de $K s$ obtidos com o ERA40 para o verão, onde a latitude crítica situa-se do equador até $20^{\circ} \mathrm{S}$ e se estende até a longitude de 180o, tendo ainda um núcleo desta latitude crítica no setor tropical da América do Sul, devido à presença de uma circulação Anticiclônica em altos níveis que favorece ventos de leste nesta região. Em torno de $30^{\circ} \mathrm{S}$ são encontrados máximos locais de Ks com valores de 6 e 7. Esta região contem valores relativamente uniformes de Ks tendo ao sul valores menores e ao norte valores mais altos, embora este último seja fruto da presença da latitude crítica e não necessariamente um menor gradiente. Entre 50 e $60^{\circ} \mathrm{S}$ é encontrado um setor uniforme de Ks com números de onda 2 e 3, e ao sul do oceano Índico em $65^{\circ} S$, onde $\beta^{*}$ e Ks são zero, ocorre a inibição da propagação das ondas de Rossby. O campo espacial de Ks para o 3MES (Fig.5b) é semelhante ao ERA40 tendo os mesmos números de onda em torno de $30^{\circ} \mathrm{S}$ e em latitudes mais altas, no entanto a área que abrange a latitude crítica sobre sul da América do Sul é menor que a obtida pelo ERA40. Nota-se também que os campos espaciais do número de onda zonal encontram-se mais suavizados tendo pequenas variabilidades espaciais com relação ao ERA40, o qual ficará mais claro nos perfis meridional do Ks.

As Figuras 6a, 6b, 6c e 6d correspondem aos perfis meridionais em $120 \mathrm{oE}, 180 \mathrm{o}, 120 \mathrm{oW}$ e $65 \mathrm{oW}$ para o período de verão onde a cor preta representa o valor obtido para o ERA40, e as outras cores representam os respectivos membros. Os membros do $3 \mathrm{MES}$ são semelhantes nos perfis meridionais em $120^{\circ} \mathrm{E}$ e em $180^{\circ}$, concordando com o ERA40, no entanto, existem algumas diferenças no setor onde existe a latitude crítica ao redor de $20^{\circ} \mathrm{S}$ e apresenta um deslocamento dos máximos de números de onda. Pode-se observar no ERA40 um máximo primário em $30^{\circ} \mathrm{S}$ o qual permite a existência de números de onda 6 e 7, e um máximo secundário ao redor de $50^{\circ} S$, favorável aos números de onda 3 e 4 , embora, em $180^{\circ}$ o modelo desloca para o norte estes máximos, mas consegue reproduzir os números de onda presentes. Para o ERA40 em $120^{\circ} \mathrm{W}$ são encontrados dois máximos, um deles em $10^{\circ} \mathrm{S}$ o qual permite números de onda 8 e 9 , e outro núcleo em $30^{\circ} \mathrm{S}$ que permite número de onda 6 , sendo bem representada 3MES. Em $65^{\circ} \mathrm{W}$ para o ERA40 pode-se observar novamente dois máximos, um ao redor de $30^{\circ} \mathrm{S}$ com número de onda 6 e outro secundário em $50^{\circ} \mathrm{S}$ com número de onda 3 . Os membros da $3 \mathrm{MES}$ reproduzem estes números de onda, mas não a variabilidade espacial dos perfis meridionais, e no setor dos trópicos onde é atingida a latitude crítica, 


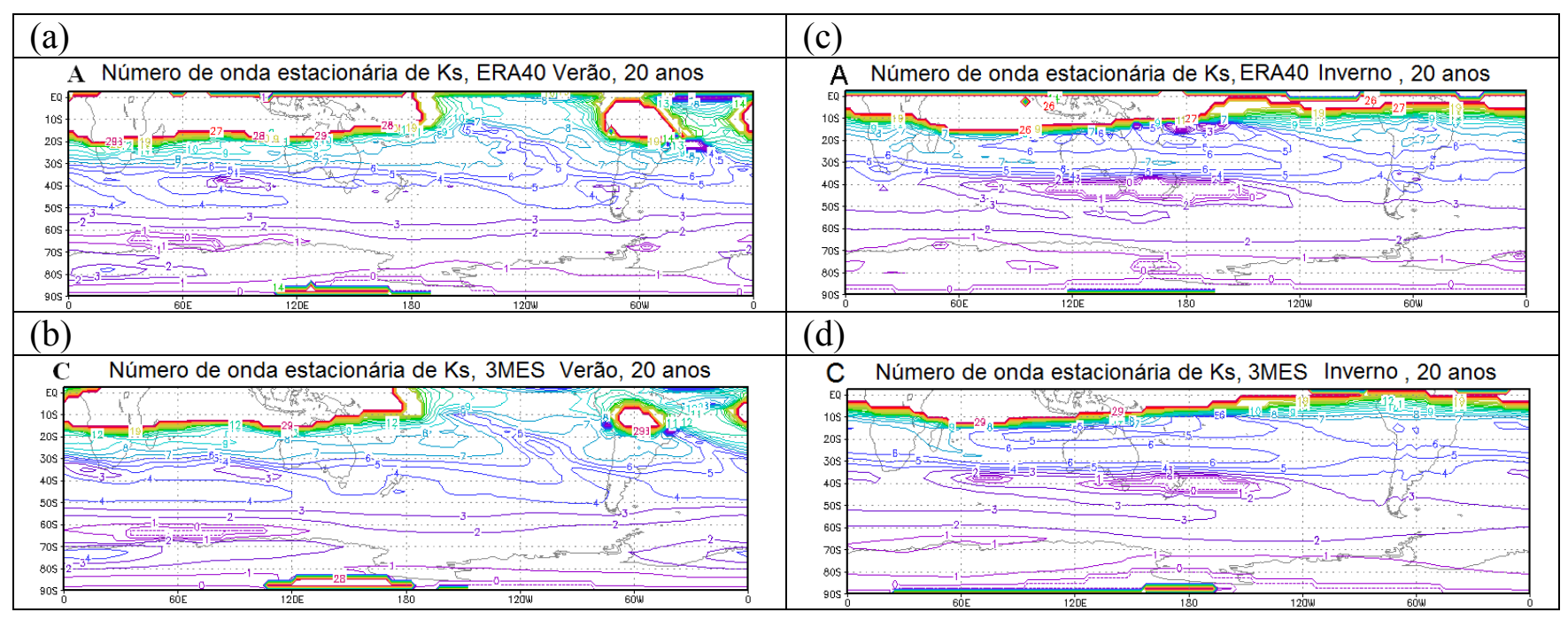

Figura 5: Média sazonal de 20 anos do Ks para o verão (a) e o inverno (c) usando o ERA40; e (b) e (d) para o 3MES. A representação dos contornos são: $(U \leq 0)$ linha grossa, $\beta^{*}$ e Ks zero (mas $U$ positivo) para linha pontilhada.

desloca-se para o equador.

Em resumo, para o verão as ondas longas (Ks entre 1 e 2) ficam confinadas entre o polo e $60^{\circ} \mathrm{S}$ (50ㅇ); ondas de número de onda zonal 3 e 4 podem-se propagar até $50^{\circ} \mathrm{S}\left(40^{\circ} \mathrm{S}\right.$ ), enquanto que as ondas mais curtas (número de onda zonal entre 5 e 8 ) podem se propagar até $25^{\circ}$ S. Ondas muito curtas (número de onda zonal acima de 8) podem se propagar através do equador, tanto para os dados da ERA40 como para cada membro da previsão sazonal.

No período de inverno os campos espaciais obtidos pelo ERA40 (Fig.5c) mostram uma latitude crítica que se aproxima a $20^{\circ} \mathrm{S}$ no setor tropical da Indonésia e diminui no Oceano Pacífico para em torno dos $10^{\circ}$ S. São observados números de onda 5 e 6 ao redor de $30^{\circ} S$ e uma região onde a propagação é inibida ao sul da Austrália (linha pontilhada) que abrange uma extensão de $130^{\circ} \mathrm{E}$ até $140^{\circ} \mathrm{W}$. Em $60^{\circ} \mathrm{S}$ nota-se uma região com possível propagação de ondas com número de onda 2 , e no continente Sul Americano existem de norte a sul números de onda que vão de 9 até 3 . O comportamento médio dos membros para os conjuntos de dados do 3MES é muito semelhante. A latitude crítica obtida pelo ERA40 consegue ser bem representada pela previsão do modelo, o mesmo acontece ao norte de $30^{\circ} \mathrm{S}$ onde são observados números de onda em torno de 5 e 6, e em $60^{\circ} \mathrm{S}$ o número de onda 2 é o predominante. Uma das diferenças mais significativas entre o modelo e os dados do ERA40 ocorre no setor em que a propagação é inibida, pois o gradiente meridional de vorticidade absoluta é zero, ou seja, ao sul da Austrália, sendo que a extensão obtida nos campos do 3MES é menor, variando de $170^{\circ} \mathrm{E}$ até $150^{\circ} \mathrm{W}$, o que certamente irá afetar o padrão de propagação de ondas nesta região.

Nas análises dos perfis meridionais do Ks obtidos com os dados do ERA40 na Fig. 6e foram encontrados em $120^{\circ} \mathrm{E}$ números de onda 6 e 7 no máximo primário o qual se encontra em $30^{\circ} S$, onde está presente a máxima intensidade do jato subtropical no inverno. Em 40ํㅗ nota-se uma região crítica onde a propagação é inibida. Em torno de 55ํㅗ é encontrado um máximo secundário mostrando número de onda 3 , uma característica que tem sido associada nesta região com casos de bloqueio no HS (TRENBERTH e MO, 1985) e considerado como um dos principais regimes de circulação para o HS (HANSEN e SUTERA, 1991). O comportamento dos membros do 3MES é semelhante, tendo diferenças nas latitudes onde atingem os máximos dos números de onda, onde os perfis meridionais são deslocados para o norte. Em 180ํㅜ (Fig.6f) acontece uma situação similar a de $120^{\circ} \mathrm{E}$, ou seja, os membros deslocam para o norte os máximos de números de onda, os valores de 6 e 7 em $30^{\circ} \mathrm{S}$ (máximo primário) e 3 em $50^{\circ} \mathrm{S}$ (máximo secundário), a diferença maior entre o modelo e o ERA40 é no setor tropical em torno de 15응 onde foi observado um gradiente meridional da vorticidade absoluta zero, sendo que os membros não reproduziram este resultado. Os perfis em 120oW e 65oW (Figs.6g, g) são semelhantes ao ERA40 e possuem os mesmos deslocamentos para norte discutidos anteriormente.

Através das análises dos campos de Ks e perfis meridionais em algumas longitudes, vimos que a previsão sazonal dos possíveis guias de onda apesar de semelhante aos dados da reanálise possui pequenos deslocamentos latitudinais, o que irá afetar o posicionamento dos guias de onda e, consequentemente, a propagação das ondas de Rossby.

\subsection{Análise do Ray Tracing (Traçado de raios)}

A Fig.7 mostra o traçado dos raios para os números de onda 2, 3 e 4 considerando as estações de verão e inverno e os dados da previsão sazonal (3MES). Os cálculos foram baseados na teoria descrita na seção b.2.2. De 


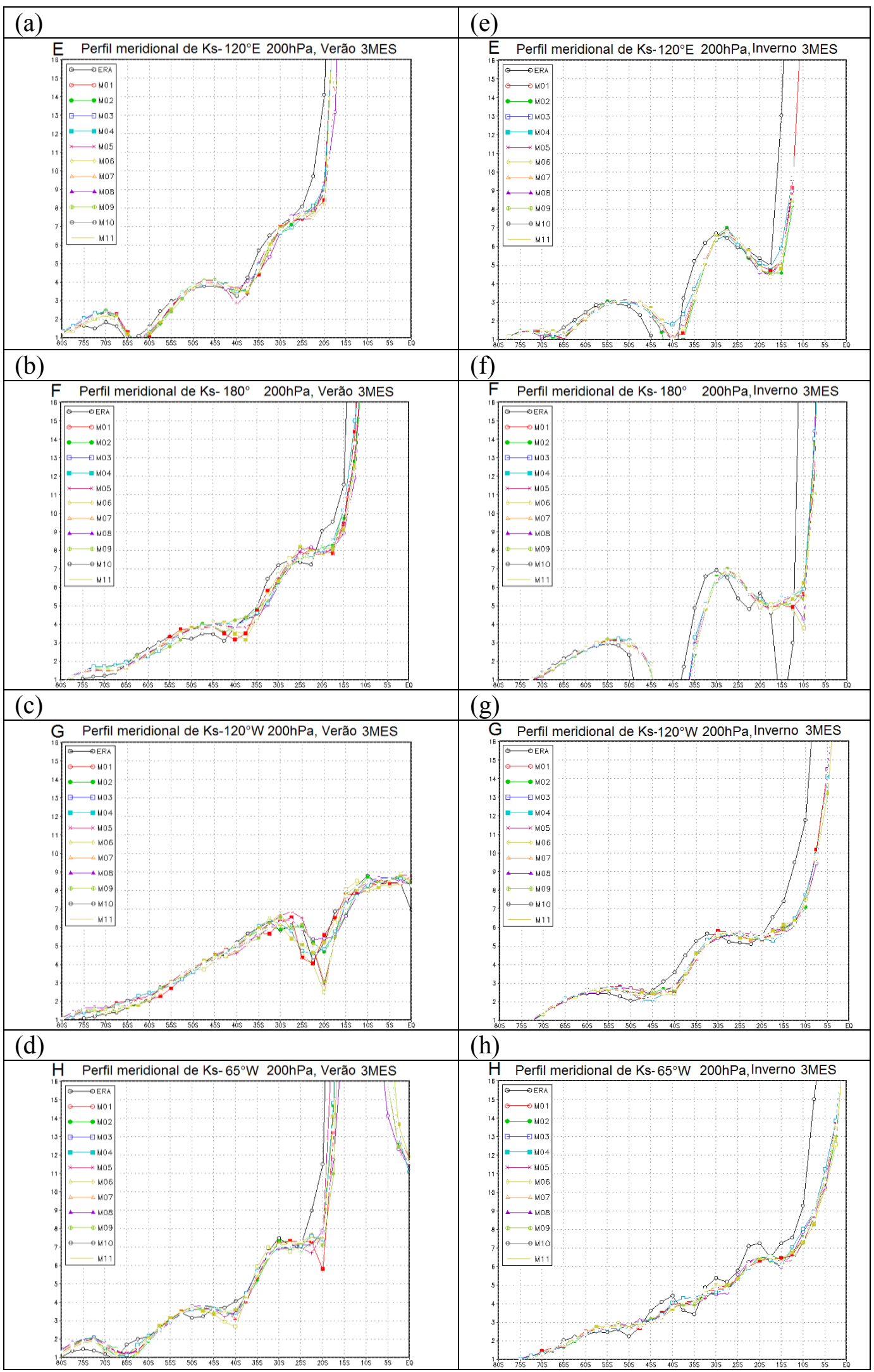

Figura 6: - Perfil meridional de Ks do 3MES em: (a, e) 120ㅌ; (b, f) 180; (c, g) 120W; (d, h) 65ํW. Figuras da esquerda para o verão e na direita inverno. A cor preta representa o ERA40 e as outras cores mostram o comportamento dos membros para a média sazonal. 

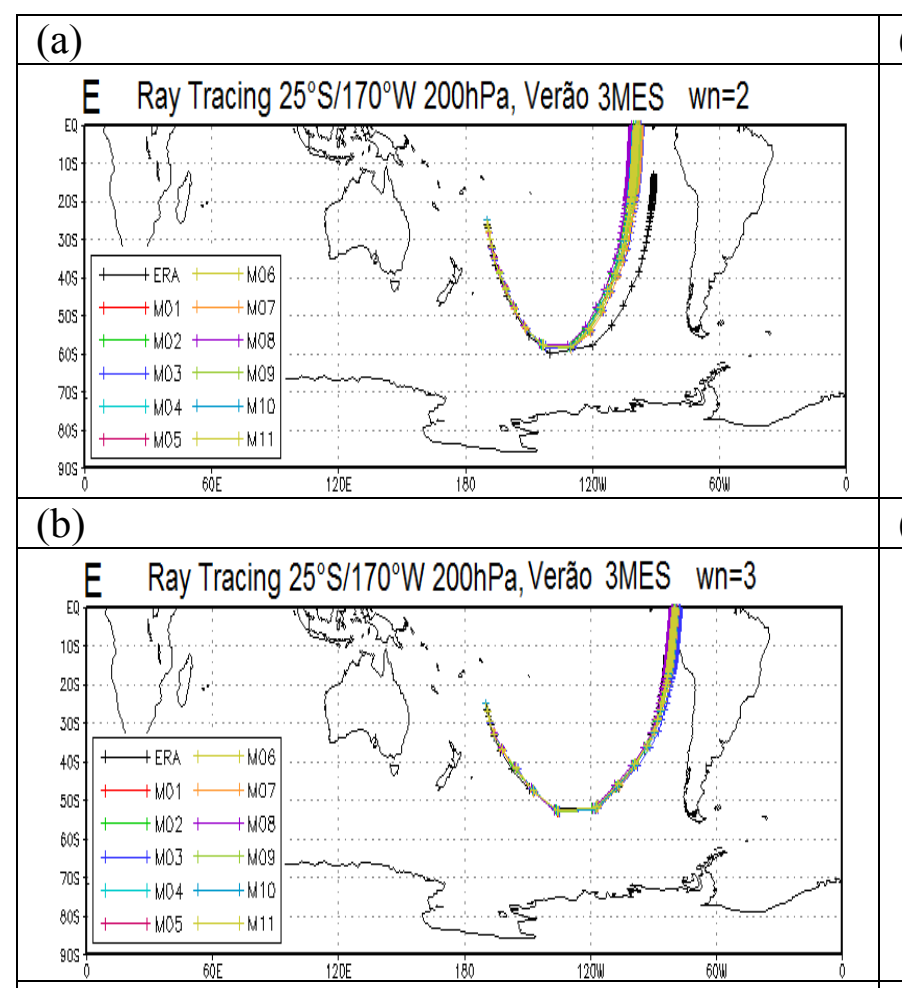

(c)

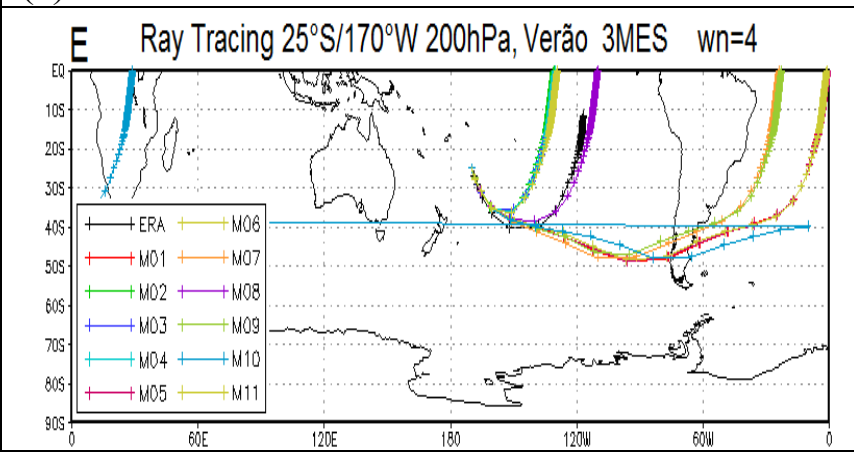

(e)

(d)
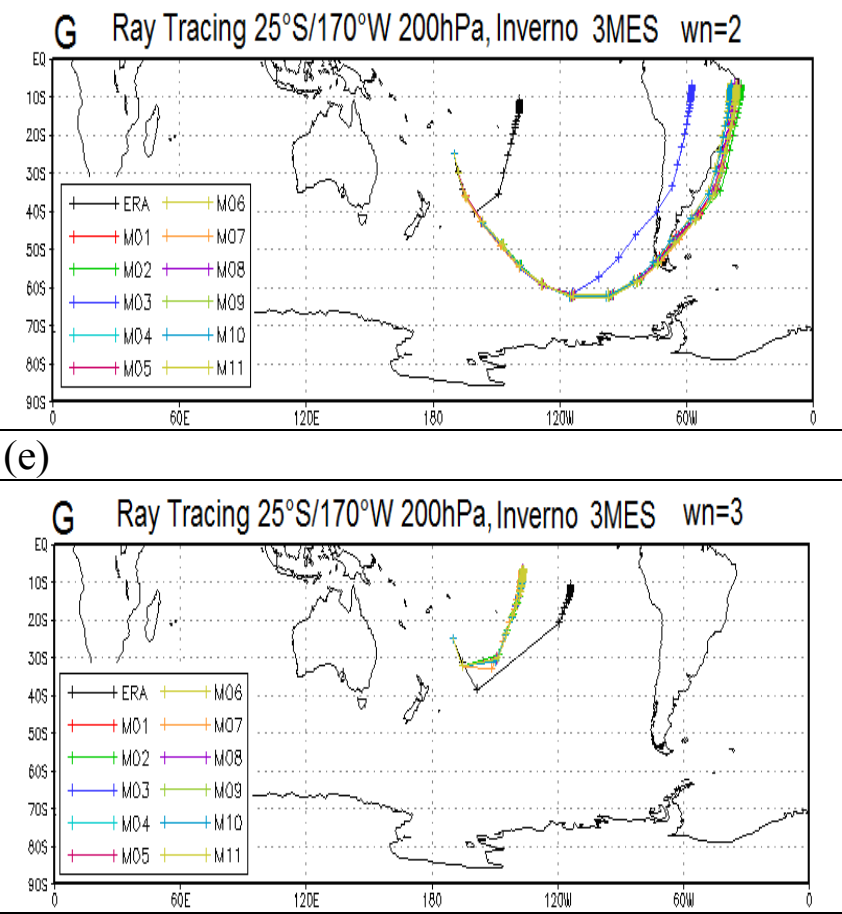

(f)

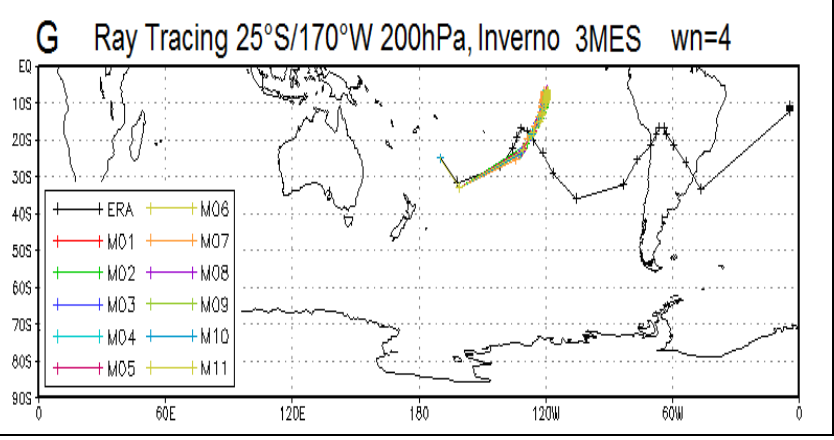

Figura 7: Ray tracing utilizando os dados do 3MES para o números de onda 2 (a, d), 3 (b, e) e 4 (c, f) considerando uma fonte em $25^{\circ} \mathrm{S}-170^{\circ} \mathrm{W}$. A cor preta representa a trajetória descrita pelo ERA40 e nas outras cores os 11 membros. Figuras da esquerda correspondem verão e da direita inverno.

forma geral, conforme já esperado pela teoria de ondas, as ondas com comprimento maior (menor número de onda) conseguem se propagar para mais altas latitudes e desta forma, a latitude crítica, que implica no ponto de retorno da onda, é mais ao sul do que para propagações com número de onda maior. Este padrão é claramente observado nas trajetórias descritas na Fig.7 tanto para o verão como para o inverno.

Durante a estação de verão, as trajetórias para os números de onda 2 e 3 tanto para o ERA40 como para o 3MES são muito próximas (Figs. 7a,b). No entanto, para o número de onda 4 (Fig.7c) (ondas mais curtas) a concordância entre os membros do modelo e a reanálise é bem menor, havendo muita dispersão entre eles. Por outro lado, as trajetórias descritas pelo modelo e ERA40 durante o inverno são mais discordantes, tanto para os números de onda pequenos quanto para os grandes (Figs. $7 \mathrm{~d}, \mathrm{e}, \mathrm{f})$. As pequenas diferenças detectadas nos campos de Ks (Fig.5) e mesmo durante seus perfis meridionais (Fig.6) são exaltadas nas análises de ray tracing, indicando que o modelo de previsão sazonal precisa melhorar sua representação dos campos básicos da atmosfera para melhorar seu skill de previsão.

\section{Discussões finais}

Este estudo avaliou o desempenho do modelo ECMWF (Sys-3) em simular o estado básico da atmosfera no Hemisfério Sul (HS) através de testes dinâmicos. A fim de determinar a habilidade do modelo e suas falhas na previsão, analisou-se diferentes estações do ano (verão 
e inverno) e a reprodução dos padrões de teleconexão atmosférica que poderiam influenciar a previsibilidade da chuva. A previsão sazonal do modelo do ECMWF foi comparada com a reanálise ERA40 do centro europeu.

Ao analisar a previsão sazonal do vento zonal no Hemisfério Sul, foi estimado que o modelo consegue representar realisticamente os principais campos espaciais, no entanto, algumas diferenças foram encontradas na variabilidade do fluxo médio zonal, havendo um deslocamento dos picos de máximos e mínimos para leste, além de não posicionar a entrada do jato polar sobre o continente Sul Americano, o que pode afetar a previsibilidade das chuvas na região Sul no período de verão e inverno. O viés sazonal encontrado entre os conjuntos de dados mostrou que a principal diferença observada ocorre nos flancos norte e sul da posição do jato subtropical e polar, subestimando também os ventos sobre o Peru e a Amazônia. Esta variabilidade do vento zonal afeta a habilidade do modelo em reproduzir o estado básico da atmosfera.

Os campos do número de onda estacionário $(K s)$ do modelo mostram se mais suavizados em relação ao observado, e falham na representação da latitude crítica sobre o continente Sul Americano, o qual abrange uma área de menor extensão deslocando-a para o norte. Essas pequenas diferenças afetam a propagação de ondas de Rossby em termos de sua trajetória na atmosfera, em particular no inverno o modelo não representou a propagação das ondas próximas ao sudeste da Austrália de forma realista. No entanto, através da análise do perfil meridional dos campos espaciais, foi encontrado que o modelo reproduz os números de onda na atmosfera e a posição dos máximos primários e secundários, permitindo, desta maneira, a propagação de ondas de Rossby, entretanto, sobre AS há pequenas variações entre os membros do modelo e o valor padrão, o que pode afetar significativamente a posição destas ondas.

Da análise do ray tracing os resultados indicam que o número de onda 2, durante o inverno (JJA) não concorda com a latitude de retorno $\left(40^{\circ} \mathrm{S}\right)$ apresentada no cálculo com a reanálise. Para o número de onda 3 no verão, os 11 membros do ECMWF conseguem seguir o traçado de raio descrito pelo ERA40, porém no inverno a variabilidade é grande, o que também é observado para o número de onda 4 . As diferenças obtidas no ray tracing demonstram que o modelo não é capaz de reproduzir corretamente o estado médio básico da atmosfera na previsão sazonal. Dependendo da época ou do comprimento de onda o impacto da propagação remota pode ser muito diferente, uma vez que a fase e a energia das ondas estão deslocadas em relação à realidade (dados observacionais) e, portanto, a possibilidade de ocorrer chuva ou não em uma região específica (previsão) pode ser comprometida.

Este estudo utilizou somente um modelo para avaliar sua previsão sazonal e trabalhou com médias de vinte anos. Obviamente, a previsão para uma determinada estação ou ano pode variar bastante ao longo do período considerado. Estudos similares devem ser realizados com outros modelos de previsão climática globais, inclusive o modelo de previsão sazonal do CPTEC/INPE (Centro de Previsão de Tempo e Estudos Climáticos - Instituto Nacional de Pesquisas Espaciais), a fim de avaliar seus erros e trabalhar para aumentar sua habilidade ("skill") de previsão sobre o Brasil mais especificadamente.

\section{Agradecimentos}

TA agradece o suporte da FAPESP (Proc. No. 08/581019) e CNPq - INCT-MC. Os resultados apresentados aqui fazem parte da Dissertação de Mestrado do Sr. Marcelo A. L. Sacco defendida junto ao Departamento de Ciências Atmosféricas do IAG/USP.

\section{Referências}

ACEITUNO, P. On the functioning of the Southern Oscillation in the South America Sector Part I: Surface Climate. Monthly Weather Review, v. 116, p. 505-524, 1988.

AMBRIZZI, T.; SOUZA, E. B.; PULWARTY, R. S. The Hadley and Walker regional circulations and associated ENSO impacts on the South American Seasonal Rainfall. In: Henry F. Diaz; Raymond S. Bradley. (Org.). The Hadley Circulation: Present, Past and Future. 1ed. Netherlands: Kluwer Academic Publishers, 2004, v. 21, p. 203-235.

AMBRIZZI, T.; HOSKINS, B. J.; HSU, H.-H. Rossby wave propagation and teleconnection patterns in the austral winter. Journal of the Atmospheric Sciences, 52, 3661-3672, 1995.

AMBRIZZI, T.; SOUZA, E. B.; PULWARTY, R. S. The Hadley and Walker regional circulations and Associated ENSO impacts on the South American Seasonal Rainfall. Capítulo do livro "The Hadley Circulation: Present, Past and Future" Edited by Henry F. Diaz and Raymond S. Bradley, Kluwer Academic Publishers, Chapter 7, 203-235, 2004.

ANDERSON, D. L.T.; STOCKDALE, T.; BALMASEDA, M.; FERRANTI, L.; VITART, F.; MOLTENI, F.; DOBLAS-REYES, F.; MOGENSON, K.; VIDARD, A. Development of the ECMWF seasonal forecast System 3. Technical Memorandum 503, 2007.

BERBERY, E. H.; NOGUES-PAEGLE, J.; HOREL, J. D. Wavelike Southern Hemisphere extratropical teleconnetions. Journal of the Atmospheric 
Sciences, v. 49, p. 155-177, 1992.

Blackmon, M. L.; Lee, Y.-H.; Wallace, J. M. Horizontal structure of $500 \mathrm{mb}$ height fluctuations with long, intermediate and short time scales. Journal of the Atmospheric Sciences, v. 41, p. 961-979, 1984.

CAVALCANTI, I. F. A.; AMBRIZZI, T. Teleconexões e suas Influências no Brasil. In: Iracema Fonseca de Albuquerque Cavalcanti; Nelson Jesus Ferreira; Maria Gertrudes Alvarez Justi da Silva; Maria Assunção Faus da Silva Dias. (Org.). Tempo e Clima no Brasil. São Paulo: Oficina de Textos, p. 317-335, 2009.

CAVALCANTI, I. F. A.; MARENGO, J. A.; SATYAMURTY, P.; NOBRE, C. A.; TROSNIKOV, I.; BONATTI, J. P.; MANZI, A. O.; TARASOVA, T.; PEZZI, L. P.; ALMEIDA, C.; SAMPAIO, D G.; CASTRO, C. C.; SANCHES, M. B.; E CAMARGO, H. Global climatological features in a simulation using the CPTEC-COLA AGCM. Journal of Climate, 15(21), 2965-2988, 2002.

COELHO C. A. S.; STEPHENSON, D. B.; DOBLASREYES, F. J.; BALMASEDA, M. From multi-model ensemble predictions to well-calibrated probability forecasts: Seasonal rainfall forecasts over South America 1959-2001. CLIVAR Exchanges No.32. v. 10, No. 1, 14-20, 2005.

COELHO C. A. S.; STEPHENSON, D. B.; BALMASEDA, M.; DOBLAS-REYES, F. J.; VAN OLDENBORGH, G. J. Toward an integrated seasonal forecasting system for South America. Journal of Climate, v. 19, p. 3704-3721, 2006.

COELHO, C. A. S. Forecast calibration and combination: Bayesian assimilation of seasonal climate predictions. Ph.D. Thesis, Reading University, 2005.

Drumond, A. R. M.; Ambrizzi, T. The role of SST on the South American atmospheric circulation observed during the 2000/2001 Austral Summer. Climate Dynamics, v. 24, 781-791, 2005.

FOLLAND, C. K.; COLMAN, A. W.; ROWELL, D. P.; DAVEY, M. K. Predictability of northeast Brazil rainfall and real-time forecast skill, 1987-98. Journal of Climate, 14(9), p. 1937- 1958, 2001.

FREITAS, A. C. V.; AMBRIZZI, T. Changes in the Austral Winter Hadley Circulation and the Impact on Stationary Rossby Waves Propagation. Advances in Meteorology, v. 2012, p. 1-15, 2012.
GRIMM, A. M.; BARROS, V. R.; DOYLE, M. E. Climate variability in Southern South America associated with El Niño and La Niña events. Journal of Climate, v. 13, 35-58, 2000.

GRIMM, A. M.; AMBRIZZI, T. Teleconnections into South America from the Tropics and Extratropics on Interannual and Intraseasonal Timescales. In: Françoise Vimeux; Florence Sylvestre; Myriam Khodri. (Org.). Past Climate Variability in South America and Surrounding Regions. Netherlands: Springer Netherlands, 2009, v. 14, p. 159-191.

GRIMM, A. M.; S. E. T. FERRAZ; J. GOMES. Precipitation anomalies in southern Brazil associated with El Niño and La Niña events. Journal of Climate, v. 11, 2863-2880, 1998.

HANSEN, A.; SUTERA, A. Planetary-scale flow regimes in midlatitudes of the Southern Hemisphere. Journal of the Atmospheric Sciences, v. 48, p. 952-964, 1991.

HOREL, J. D.; WALLACE, J. M. Planetary-scale atmospheric phenomena associated with the Southern Oscilllation. Monthly Weather Review, v. 109, 813-829, 1981.

HOSKINS, B. J.; AMBRIZZI, T. Rossby wave propagation on a realistic longitudinally varying flow. Journal of the Atmospheric Sciences, v. 50, 1661-1671, 1993.

HOSKINS, B.J.; KAROLY, D. J. The steady linear response of a spherical atmosphere to thermal and orographic forcing, Journal of the Atmospheric Sciences, v. 38, p. 1179-1196, 1981.

HSU, H. -H.; LIN, S.-H. Global teleconnections in the $250 \mathrm{mb}$ streamfunction field during the Northern Hemisphere winter. Monthly Weather Review, v. 120, 1169-1190, 1992.

KAROLY, D. J. Rossby wave propagation in a barotropic atmosphere. Dynamics of Atmospheres and Oceans, v. 7, p. 111-125, 1983.

KILADIS, G. N.; Diaz, H. F. Global climatic anomalies associated with extremes in the Southern Oscillation. Journal of Climate, v. 2, p. 1069-1090, 1989.

KOUSKY V. E.; KAGANO, M. T.; CAVALCANTI, I. F. A. A review of the Southern Oscillation: oceanicatmospheric circulation changes and related rainfall anomalies. Tellus, v. 36A, p. 490-504, 1984. 
MAGAÑA, V.; AMBRIZZI, T. Dynamics of subtropical vertical motions over the Americas during El Niño boreal winters. Atmósfera, 18(4), p. 211-233, 2005.

MARENGO J. A.; CHOU, S. C.; KAY, G.; ALVES, L. M.; PESQUERO, J. F.; SOARES, W. R.; SANTOS, D. C.; LYRA, A. A.; SUEIRO, G.; BETTS, R.; CHAGAS, D. J.; GOMES, J. L.; BUSTAMANTE, J. F.; TAVARES, P. Development of regional future climate change scenarios in South America using the Eta CPTEC/HadCM3 climate change projections: climatology and regional analyses for the Amazon, São Francisco and the Parana River basins. Climate Dynamics, v. 23, p. 1-20, 2011.

MARENGO, J.; CAVALCANTI, I. F. A.; SATYAMURTY, P.; TROSNIKOV, I.; NOBRE, C. A.; BONATTI, J. P.; CAMARGO, H.; SAMPAIO, G.; SANCHES, M. B.; MANZI, A.; CASTRO, C. A. C.; ALMEIDA, C. D.; PEZZI, L. P.; CANDIDO, L. Assessment of regional seasonal rainfall predictability using CPTEC/COLA atmospheric GCM. Climate Dynamics, 21(5-6), p. 459-475, 2003.

MOURA, A. D.; HASTENRATH, S. Climate prediction for Brazil's Nordeste: Performance of empirical and numerical modeling methods. Journal of Climate, 17(13), p. 2667-2672, 2004.

PEZZI, L. P.; CAVALCANTI, I. F. A. The relative importance of ENSO and tropical Atlantic sea surface temperature anomalies for seasonal precipitation over South America: a numerical study. Climate Dynamics, v. 17, p. 205-212, 2001.

RAO, V. B.; HADA, K. Characteristics of rainfall over Brazil: Annual variations and connections with Southern Oscillation. Theoretical and Applied Climatology, v. 42, p. 81-91, 1990.

ROPELEWSKI, C. F.; HALPERT, M. S. North American precipitation and temperature associated with the El Niño Southern Oscillation (ENSO). Monthly Weather Review, v. 114, p. 2352-2362, 1986.

ROPELEWSKI, C. F.; HALPERT, M. S. Global and regional scale precipitation patterns associated with El Niño/Southern Oscillation. Monthly Weather Review, v. 115, p. 1606-1626, 1987.

ROPELEWSKI, C. F.; HALPERT, M. S. Precipitation patterns associated with high index phase of Southern Oscillation. Journal of Climate, v. 2, p. 268-284, 1989.
STOECKENIUS, T. Interannual variations of tropical precipitation patterns. Monthly Weather Review, v. 109, p. 1233-1247, 1981.

TRENBERTH, K.E.; MO, K.C. Blocking in the Southern Hemisphere. Monthly Weather Review, v. 113, p. 3-21, 1985.

TRENBERTH, K. E.; BRANSTATOR, G. W.; KAROLY, D.; KUMAR, A.; LAU, N.-C.; ROPELEWSKI, C. Progress during TOGA in understanding and modeling global teleconnections associated with tropical sea surface temperatures. Journal of Geophysical Research, v. 103, C7, p. 14291-14324, 1998.

UVO, C. R. B.; REPELLI, C. A.; ZEBIAK, S. E.; KUSHNIR, Y. The relationships between tropical Pacific and Atlantic SST and northeast Brazil monthly precipitation. Journal of Climate, v. 11, p. 551-562, 1998.

WALLACE, J. M.; GUTZLER, D. S. Teleconections in the geopotential height field during the Northern Hemisphere winter. Monthly Weather Review, v. 109, p. 785-812, 1981.

YANG, G. -Y.; HOSKINS, B. J. Propagation of Rossby waves of nonzero frequency. Journal of the Atmospheric Sciences, v. 53, p. 2365-2378, 1996. 